\title{
VOM VERSUCH, JOHANN KALO, DEN PFARRER DER BARTHOLOMÄUSPFARRKIRCHE IN DER JUNGSTADT DANZIG, MIT DEM ERMLÄNDISCHEN DOMHERRN JOHANN KALLE GLEICHZUSETZEN \\ UND EINIGE ANDERE RANDBEMERKUNGEN ZUM NEUESTEN BUCH VON PIOTR SAMÓL*
}

Piotr Samól, Młode Miasto Gdańsk (1380-1455) i jego patrymonium, (2018).

Der vorliegende Beitrag entstand im Zusammenhang mit dem neu erschienenen Buch von Piotr Samól über die Jungstadt Danzig. Im Vorhinein möchte ich hervorheben, dass ich die Arbeit des Danziger Forschers sehr hochschätze und meine Randbemerkungen grundsätzlich weder den Wert der Publikation herabsetzen noch die in der Monografie aufgenommenen Hauptfragen bestreiten. Da aber der Autor in seinem Buch den Versuch unternahm, den Pfarrer der Bartholomäuspfarrkirche in der Jungstadt Danzig mit einem ermländischen Domherrn gleichzusetzen, beschloss ich, die Gelegenheit zu nutzen, um das Wort zu ergreifen und einen Ausschnitt meiner neuesten Untersuchungen zur personellen Zusammensetzung des

* Der Beitrag entstand im Zusammenhang mit dem Projekt Prozopografia duchowieństwa diecezji warmińskiej w średniowieczu (Prosopographie der Geistlichkeit des Bistums Ermland im Mittelalter), das aus den Mitteln des Nationalen Wissenschaftszentrums (Narodowe Centrum Nauki) aufgrund des Vertrags Nr. DEC-2011/03/N/HS3/01145 finanziert wurde.

1 Die Jungstadt Danzig und ihre Besitzungen. 
ermländischen Domkapitels im Mittelalter darzustellen². Meine Absicht ist ebenfalls, auf einige andere Fragen hinzuweisen, die sich auf die rezensierte Monografie beziehen, sowie den Buchinhalt näher zu schildern. Dadurch will ich andere Forscher anspornen, sich mit den unumstritten wichtigen, von Piotr Samól im Buch angeführten Feststellungen vertraut zu machen.

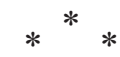

Das besagte Buch erschien 2018 im Verlag der Universität Danzig. Zu Rezensenten wurden Prof. Dr. Janusz Tandecki von der Nikolaus-Kopernikus-Universität in Toruń und Prof. Dr. Joachim Zdrenka von der Universität Zielona Góra berufen. Die Grundlage dieser Veröffentlichung bildete eine Dissertation, die an der Historischen Fakultät der Universität Danzig, unter Betreuung von Prof. Beata Możejko, vorbereitet wurde. Dem Autor zufolge wurden die Untersuchungen zur besagten Thematik in einem Zeitraum von acht Jahren durchgeführt, während deren er die Forschungsmaterialien sowohl in Polen als auch im Ausland sammelte.

Die Publikation beginnt mit einem Vorwort (S. 9-10), dem eine Einleitung (S. 11-35) folgt. Der Autor schildert darin das Themenfeld seiner Untersuchungen sowie den chronologischen Rahmen. Erwähnenswert ist, dass das Buch von P. Samól die ganze Zeitspanne umfasst, in der die Jungstadt Danzig und ihr patrimonium (Besitzungen der Stadt) bestanden, und zwar angefangen mit der Gründung der Stadt 1380 bis zu ihrer Auflösung 1455. Somit wird eine Lücke in der Geschichtsschreibung gefüllt, zumal der Autor eine Reihe von detaillierten, grundlegenden Feststellungen trifft, u. a. in Bezug auf die Raumentwicklung Danzigs, die Entstehung und schließlich auch den Untergang der Jungstadt, die personelle Zusammensetzung der Stadtregierung und die Kirchenorganisation dieser Stadt, aber auch auf die sozialen und wirtschaftlichen Beziehungen ihrer Einwohner. In der Einleitung analysiert er den Forschungsstand, fasst ihn zusammen und bestimmt ihn zu Recht als „allzu unbefriedigend“ (S. 23). Er weist auch auf ein „Durcheinander“ in den bisherigen populärwissenschaftlichen

2 Mehr Angaben dazu in einer gerade zum Druck vorbereiteten Monografie: R. Krajniak, Prałaci i kanonicy warmińskiej kapituły katedralnej do 1466 roku. Studium prozopograficzne. 
Arbeiten hin, besonders in zahlreichen Veröffentlichungen, die von nicht sachverständigen Personen herausgegeben werden. Ferner legt Samól die Quellengrundlage der Arbeit dar und macht u. a. wichtige Bemerkungen über die Kanzlei der Jungstadt und ihre Überlieferung. Der Autor deutet auch darauf hin, dass die Ordensurkunden und andere Danziger Zeugnisse, aufgrund deren er seine Monografie bearbeitete, angesichts relativ weniger ausschließlich jungstädtischer Quellen einen besonderen Wert haben. Er erläutert also die in der Arbeit genutzten handschriftlichen Quellen aus dem Bestand des Staatsarchivs in Danzig und des Geheimen Staatsarchivs Preußischer Kulturbesitz in Berlin sowie die gedruckten Quellen und weist auf die von ihm genutzten kartografischen Quellen aus dem Bestand des Danziger Archivs und der Danziger Bibliothek der Polnischen Akademie der Wissenschaften hin. Anschließend informiert er über die angewandten Forschungsmethoden und stellt fest, dass sie zwar zu den Geschichtswissenschaften gehören, aber um die für die technischen Wissenschaften typischen Methoden ergänzt wurden (S. 31).

Das erste Buchkapitel (die einzelnen Teile der Arbeit werden aber als Kapitel nicht bezeichnet) erhielt den Titel Geneza powstania, przestrzeń i ustrój Młodego Miasta (Die Entstehungsgenese, der Raum und das Stadtsystem der Jungstadt, S. 37-150). Es fängt mit den Erwägungen über die Entstehung der Stadt und über die Zeitspanne vor diesem Ereignis an. Der Autor behauptet darin u. a., dass die Jungstadt als einzige der Danziger Stadtgemeinden aus wilder Wurzel angelegt wurde. In diesem Abschnitt schildert er auch die Finanzeinkünfte des Deutschen Ordens aus der Jungstadt. Der zweite (in der Arbeit nicht nummerierte) Teil des genannten Kapitels rekurriert auf den Stadtraum. Hier bemüht sich der Autor, den Siedlungsraum sowie die Grenze zwischen der Alt- und der Jungstadt zu bestimmen und das Stadtplanungsprojekt darzustellen. Er bespricht auch die Entstehung der Anlegestelle (des Hafens) - des Bollwerks - und charakterisiert das patrimonium der Jungstadt. Dieser Teil des Kapitels wird mit besonders wertvollen Zeichnungen des Autors selbst abgeschlossen, die das Gebiet der Jungstadt in der Zeit ihres Bestehens sowie auch nach ihrer Auflösung in den folgenden Jahrhunderten zeigen. Zusätzlich wird, u. a. in einer hypothetischen Skizze, der Stadtplan der Jungstadt geschildert. $\mathrm{Zu}$ diesem Buchteil gehören auch die Bemerkungen des Autors über das Stadtsystem, darunter besonders wertvolle Feststellungen über den Rat, das Richteramt sowie das Funktionieren der städtischen Schöffenbank. Das 
Kapitel schließt mit farbigen Plänen und Ansichten vor allem aus dem 16. und 17. Jahrhundert sowie einzelnen Zeichnungen aus dem 18., 19. und 20. Jahrhundert, die das vom Autor untersuchte Gebiet zeigen.

Das zweite Buchkapitel unter dem Titel Organizacja kościelna w Młodym Mieście (Die Kirchenorganisation in der Jungstadt, S. 151-214) enthält in erster Linie Bemerkungen zur sakralen Stadttopografie. Der Autor geht hier u. a. auf die Theorien über die Anzahl der Kirchen ein, sieht die Feststellungen der bisherigen Geschichtsschreibung kritisch und analysiert die Lage des Karmeliterklosters und der Bartholomäuskirche. Der darauffolgende Teil des Kapitels präsentiert die Geschichte der einzelnen Gotteshäuser und beginnt mit Angaben über die Bartholomäuskirche und die dortigen Pfarrer. Zudem wird auch auf die Geschichte der Marienkirche und des Karmeliterklosters sowie der Spitäler von Aller Gottes Engel und von St. Jakob eingegangen. Ähnlich wie im ersten Kapitel schließt auch dieses mit farbigen Ansichten und Plänen aus der Zeit vom 17. bis zum 19. Jahrhundert, die vor allem die Kirche Aller Gottes Engel schildern.

Im dritten Buchkapitel unter dem Titel Ludzie i gospodarka (Menschen und Wirtschaft, S. 215-292) findet man zunächst Angaben zur Demografie der Jungstadt. Hier werden die ersten Versuche unternommen, die Zahl der Einwohner einzuschätzen, aber auch die Frage der Bürgerschaft in den Gemeinden der mittelalterlichen Stadt Danzig erwogen. Der Autor bemüht sich später darum, die demografischen Tendenzen in der Jungstadt zu erfassen. Im darauffolgenden Teil des Kapitels schildert er die Forschungsergebnisse zur politischen und wirtschaftlichen Stadtelite und führt chronologisch die wichtigsten jungstädtischen Familien an. Anschließend finden sich hier die Angaben zu den Einkünften der Einwohner. Es werden u. a. diejenigen Fragen untersucht, die mit der Handelstätigkeit der Einwohner verbunden sind, darunter die Handelskontakte der Stadt, das dortige Handwerk sowie der Bau- und Kapitelmarkt.

Das vierte Kapitel wurde Likwidacja Młodego Miasta (Die Auflösung der Jungstadt, S. 293-358) betitelt. P. Samól bespricht hier die Ereignisse, die dem sog. dreizehnjährigen Krieg (1454-1466) vorangingen, die erste Zerstörung der Jungstadt, die Zusammenarbeit der Stadt mit anderen Gemeinden in Danzig sowie ihr Schicksal im ersten Kriegsjahr, darunter die endgültige Stadtzerstörung. Er schildert auch das Geschehen nach der Stadtauflösung, darunter die Haltung der ehemaligen Einwohner während des dreizehnjährigen Krieges, sowie die Frage der Auflösung des jung- 
städtischen patrimoniums. Diesem Buchteil legte er die Abbildungen u. a. des Siegels der Jungstadt Danzig sowie die Ansichten und das Panorama Danzigs und der Gebiete der früheren Jungstadt aus der Zeitspanne vom 16. bis zum 20. Jahrhundert bei. Im Buch findet man auch Fotos aus den archäologischen Befunden aus dem Jahr 2018, die u. a. die Fundamente der jungstädtischen Häuser darstellen.

Der letzte Buchteil erhielt den Titel Epilog. Młode Miasto jako tzw. Miasto Nowe" (Epilog. Die Jungstadt als die sog. Neustadt, S. 359-379). P. Samól versucht hier, die Frage zu beantworten, wie die Stadtstruktur der Jungstadt Danzig in den allgemeinen Prozess der Gründung von Städten in Preußen einzuordnen ist (S. 359). Um dieses Forschungsproblem zu untersuchen, stützt er sich auf das ehemals von Zenon Hubert Nowak vorgeschlagene Formular und setzt sich mit einigen detaillierten Fragen auseinander, darunter mit der Gründung der Stadt, den Beziehungen zwischen der „neuen Stadt" und dem Deutschen Orden, dem Verhältnis zur ,alten Stadt“ und mit der Aufhebung der Unabhängigkeit der „neuen Stadt" (S. 359).

Das rezensierte Buch schließt mit der Zusammenfassung (S. 381-385), dem Abkürzungsverzeichnis (S. 387-388), den Verzeichnissen der Abbildungen (S. 389-391), Tabellen (S. 392) und Tafeln (S. 393) sowie dem Literaturverzeichnis (S. 394-416) mit der Gliederung in Archivquellen (S. 394-396), gedruckte Quellen (S. 396-399) und Literatur (S. 399-416) ab. Das Ganze ergänzen noch die einige Seiten lange Zusammenfassung in englischer Sprache (S. 417-421) sowie eine professionelle Beilage auf Pauspapier, u. a. mit den Abbildungen von den einzelnen Etappen der Raumentwicklung Danzigs und der Gebiete, wo die Jungstadt angelegt wurde. Diese Lösung, d. i. die Beilage auf Pauspapier, erlaubt, die einzelnen Entwicklungsetappen miteinander zu vergleichen.

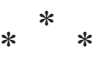

Bevor ich zu den kritischen Bemerkungen übergehe, möchte ich auf den im Titel meines Beitrags angesprochene Gleichsetzung eingehen. Johann Kalo, der Pfarrer der Bartholomäuskirche, der in diesem Amt zweifelsohne in den Jahren 1444-1445 (S. 174-175) belegt ist, wird von P. Samól mit dem ermländischen Domherrn Johann Kalle gleichgesetzt. Der Autor, der einige sehr wertvolle Angaben zur Tätigkeit des Pfarrers der jungstädtischen 
Pfarrei machte ${ }^{3}$, bemerkt, dass Johann Kalo „1447 höchstwahrscheinlich als ermländischer Domherr" starb (S. 175). Um die eigene Aussage zu bekräftigen, beruft er sich ausschließlich auf eine Urkunde päpstlicher Provenienz, aus der hervorgeht, dass Johann Kalle (der meiner Einsicht nach nicht mit dem vom Autor genannten Geistlichen zu identifizieren ist) bereits am 2. März 1448 nicht mehr lebte ${ }^{4}$. Woraus ergibt sich also die Behauptung, dass er bereits 1447 starb? Da ich es für weniger wahrscheinlich halte, dass der Pfarrer Johann Kalo mit dem ermländischen Domherrn identisch ist, möchte ich auf die Laufbahn Johann Kalles Bezug nehmen und darlegen, was wir tatsächlich vom Leben und von der Tätigkeit des zur selben Zeit lebenden ermländischen Domherrn, der einen Namen trug, welcher in seinem Stamm dem Namen des Pfarrers der Bartholomäuspfarrei ähnelt, wissen.

Johann Kalle (Calle, Kalle, Kallen) ${ }^{5}$, das spätere Mitglied des ermländischen Domkapitels, stammte aus Königsberg. Als „Joannes Kalle de Konighesberch" wird er in den Akten der Universität Prag erwähnt ${ }^{6}$. Von seiner

3 Es ist aber auf einen kleinen Fehler hinzuweisen. Auf S. 175 in Anm. 132 ist eine falsche Signatur angeführt. Anstatt Schubl. 91, Nr. 27 sollte die Signatur Schubl. 91, Nr. 28 genannt werden. Nebenbei sollte man noch auf einen Fehler hindeuten, und zwar in der Zitation von Pergamenturkunden aus dem Bestand des Geheimen Staatsarchivs Preußischer Kulturbesitz (weiter als: GStA PK). Auf S. 176 in Anm. 137 sollte anstatt der Signatur Schubl. 41, Nr. 21 die Signatur Schubl. XLI, Nr. 21 angeführt werden.

4 Siehe Bullarium Poloniae litteras apostolicas aliaque monumenta Poloniae Vaticana continens (weiter als: BP), 6: 1447-1464, hg. v. I. Sułkowska-Kuraś, S. Kuraś, (1998), Nr. 134.

5 Vgl. die früheren seiner Biogramme: T. Borawska, Kalle Jan, in: Słownik biograficzny kapituły warmińskiej, bearb. v. T. Borawska, M. Borzyszkowski, A. Kopiczko, J. Wojtkowski, hg. v. J. Guzowski, (1996), S. 108; T. Jurkowlaniec, Nagrobki średniowieczne w Prusach, (2015), S. 177.

6 Liber decanorum facultatis philosophicae Universitatis Pragensis ab anno Christi 1367 usque ad annum 1585, 1 (Monumenta historica Universitatis Carolo-Ferdinandae Pragensis 1, 1830), S. 276; siehe auch Prussia scholastica. Die Ost- und Westpreussen auf den mittelalterlichen Universitäten, gesammelt v. M. Perlbach, (1895), S. 15; J. Tříška, Životopisný slovník předhusitské pražské univerzity 1348-1409, (1981), S. 262; H. Kenkel, Studenten aus Ost- und Westpreußen an außerpreußischen Universitäten vor 1815, (1981), S. 450. Daraus ließen ihn auch die übrigen Forscher herleiten, die sich früher mit seiner Person auseinandersetzten, siehe: H. Freytag, Die Beziehungen der Universität Leipzig zu Preussen von ihrer Begründung bis zur Reformation 1409-1539, in: Zeitschrift des Westpreussischen Geschichtsvereins, 44 (1902), S. 126; Borawska, Kalle Jan, S. 108; R. Ruciński, Środowiska intelektualne wielkich miast pruskich w średniowieczu, (2005), (Manuskript der Dissertation), S. 181. 
Königsberger Herkunft zeugt auch die Inschrift auf seiner Grabplatte ${ }^{7}$. Beachtenswert ist aber, dass man auf die Personen, die sich dieses Namens bedienten, in der uns interessierenden Zeitspanne u. a. in Braunsberg stößt ${ }^{8}$. Kalle war sehr gut ausgebildet. Seit 1390 studierte er in Prag, wo er zwei Jahre später Bakkalaureus der Freien Künste wurde 9 . In der Fachliteratur setzt man ihn übrigens mit dem $1394 \mathrm{zu}$ den ungarischen Nationes an der Universität Wien immatrikulierten „Johannes de Monte Regio“ gleich ${ }^{10}$. In kurzer Zeit muss Kalle auch den Magistergrad der Freien Künste erlangt haben ${ }^{11}$. Am Rande sollte man auch darauf hindeuten - obwohl wir es in diesem Falle sicherlich mit zwei unterschiedlichen Personen zu tun haben -, dass ein weiterer Johann Kalle, der aus Elbing stammte, 1398 ein Jura-

7 Jurkowlaniec, Nagrobki, S. 177.

8 In den Quellen sind sie schon mindestens seit der zweiten Hälfte des 14. Jahrhunderts belegt, siehe u. a.: Codex diplomaticus Warmiensis oder Regesten und Urkunden zur Geschichte Ermlands (weiter als: CDW), hg. v. V. Röhrich, F. Liedtke, H. Schmauch, 4 (1935), Nr. 1, S. 3, 11, 48 und Nr. 609, S. 623. Johannes Kale war 1446-1457 Ratsherr in der Altstadt Braunsberg, siehe D. Bogdan, J. Przeracki, Urzędnicy Starego i Nowego Miasta Braniewa do 1772 roku, (2018), S. 632.

9 Liber decanorum, S. 276; Prussia scholastica, S. 15, siehe auch Tříška, Životopisný slovník, S. 262; Kenkel, Studenten, S. 450; Ruciński, Środowiska intelektualne, S. 181; vgl. auch die Bemerkungen der Autoren, die erweiterte biografische Angaben anführen: Freytag, Die Beziehungen, S. 126; Borawska, Kalle Jan, S. 108; Jurkowlaniec, Nagrobki, S. 177.

10 Siehe Die Matrikel der Universität Wien, 1: 1377-1450, (1954), S. 43. Schon Perlbach setzt diese Person zweifelsohne mit Johann Kalle gleich, siehe Prussia scholastica, S. 24, 198. Diese Information wird auch einstimmig in der Fachliteratur wiederholt, siehe Freytag, Die Beziehungen, S. 126; Borawska, Kalle Jan, S. 108; Ruciński, Środowiska intelektualne, S. 181; Jurkowlaniec, Nagrobki, S. 177. Auch Kenkel behauptet, dass Johannes de Monte Regio zum Kreis der Studenten aus Preußen gehörte, siehe idem, Studenten, S. 270.

11 Borawska, Kalle Jan, S. 108; Jurkowlaniec, Nagrobki, S. 177. Johann Kalle tritt als Magister beispielsweise schon am 21. November 1417 auf, siehe Repertorium Germanicum. Verzeichnis der in den päpstlichen Registern und Kameralakten vorkommenden Personen, Kirchen und Orte des Deutschen Reiches, seiner Diözesen und Territorien vom Beginn des Schismas bis zur Reformation (weiter als: RG), bearb. v. K. A. Fink, 4 (1943-1958), Sp. 1699. Später war er als Magister u. a. auch 1431 belegt, siehe CDW 4, Nr. 393, 13. Februar 1436, siehe GStA PK, XX. Hauptabteilung (weiter als: XX. HA), Ordensfolianten (weiter als: OF) 96, K. 84r-86r, 28. Februar 1445, siehe Archiwum Archidiecezji Warmińskiej w Olsztynie (weiter als: AAWO), Dokumenty Kapituły (weiter als: Dok. Kap.) V 17a, 14 Mai 1446, siehe AAWO, Archiwum Kapituły (weiter als: AK) I, T 7, Fol. 92 und 14. August 1447, siehe GStA PK, XX. HA, Pergament-Urkunden (weiter als: Perg. Urk.), Schublade (weiter als: Schubl.) 90 Nr. 7; siehe ebenfalls AAWO, Acta Capitularia (weiter als: Acta Cap.), S 1, K. 54v; Archiwum Biskupie (weiter als: AB), Eh 41. Der erhaltene Magistergrad wurde darüber hinaus in seine Grabplatte eingehauen, siehe Jurkowlaniec, Nagrobki, S. 177. 
studium in Prag begann ${ }^{12}$ und dort 1400 als Magister der Freien Künste belegt ist ${ }^{13}$.

Johann Kalle wurde laut Teresa Borawska am 16. August 1416 ermländischer Domherr ${ }^{14}$. Carl Peter Woelky stellt dagegen fest, dass er als Domherr schon seit dem 16. März dieses Jahres belegt is $\mathrm{t}^{15}$. Beide Angaben wurden aber mit keinen Quellenbeweisen bestätigt ${ }^{16}$. In den Urkunden päpstlicher Provenienz nannte man ihn am 13. Februar 1417 als Mitglied des Domkapitelkollegiums ${ }^{17}$. Zu diesem Zeitpunkt war er auch als Sekretär der Apostolischen Kammer belegt ${ }^{18}$. Am 21. November dieses Jahres wurde er dagegen vom Papst in dem Kanonikat und einer großen ermländischen Pfründe eingesetzt, die im Zusammenhang mit der Wahl Johann Abeziers zum ermländischen Bischof vakant geworden war. Man findet ihn dort auch als Kleriker der samländischen Diözese und Sekretär des Rigaer Erzbischofs Johann ${ }^{19}$. Zu diesem Zeitpunkt soll er darüber hinaus auch Altarist an der St.-Nikolaus-Kirche in Königsberg gewesen $\operatorname{sein}^{20}$.

12 Album seu matricula juridicae Universitatis Pragensis ab anno Christi 1372 usque ad annum 1418, 1 (Monumenta historica Universitatis Carolo-Ferdinandae Pragensis 2, 1834), S. 109; Prussia scholastica, S. 21; siehe auch Tř́ška, Životopisný slovník, S. 262; Kenkel, Studenten, S. 454; Ruciński, Środowiska intelektualne, S. 169.

13 Liber decanorum, S. 347; Prussia scholastica, S. 17; siehe auch Tř́šša, Životopisný slovník, S. 228; Kenkel, Studenten, S. 451; Ruciński, Środowiska intelektualne, S. 169.

14 Siehe Borawska, Kalle Jan, S. 108. Freytag behauptet, dass Johann in den Jahren 1416-1448 Domherr war, siehe idem, Die Beziehungen, S. 126. Seine Amtszeit in dem ermländischen Kanonikat bestimmte man gleichermaßen in: Prussia scholastica, S. 198. Zuletzt stellte auch Jurkowlaniec fest, dass Kalle das ermländische Kanonikat seit 1416 innehatte, siehe idem, Nagrobki, S. 177.

15 Scriptores rerum Warmiensium oder Quellenschriften zur Geschichte Ermlands (weiter als: SRW), hg. v. C. P. Woelky, J. M. Saage, 1 (1866), S. 245, Anm. 138.

16 Es ist sicher, dass die Jahre 1416-1448 auch in einem unveröffentlichten Verzeichnis, das sich im Bestand des Archivs des Ermländischen Erzbistums in Allenstein befindet, als die Amtszeit Kalles in dem Kanonikat bestimmt wurden, siehe AAWO, AB, H 295c.

17 RG, bearb. v. U. Kühne, 3 (1935), Sp. 392.

18 Ibid. Diese Tatsache wurde in der Geschichtsschreibung bereits wahrgenommen, siehe Borawska, Kalle Jan, S. 108; siehe auch Jurkowlaniec, Nagrobki, S. 177.

19 RG 4, Sp. 1699; siehe auch Borawska, Kalle Jan, S. 108; Jurkowlaniec, Nagrobki, S. 177.

20 RG 4, Sp. 1699. Dass er eine Altarpfründe besaß, stellte schon Borawska fest, die aber nicht präzise angibt, wann Johann tatsächlich auf dieser Pfründe belegt ist, siehe eadem, Kalle Jan, S. 108. Einen Fehler beging dagegen Jurkowlaniec, der behauptet, dass er schon seit 1416 Altarist war, siehe idem, Nagrobki, S. 177. 
T. Borawska zufolge trat Kalle in den ermländischen Urkunden am 30. März 1425 auf $^{21}$. Der Geistliche ist damals tatsächlich in Frauenburg zusammen mit anderen Domherren belegt ${ }^{22}$, doch in den lokalen Quellen schon am 11. November 1422 in Mehlsack ${ }^{23}$. Noch im selben Jahr, am 18. Dezember, erwähnte man ihn zudem in Frauenburg ${ }^{24}$. Ferner stößt man auf ihn in dieser Stadt am 8. Juli 1426, als die notariell bezeugte Abschrift der Goldenen Bulle Kaiser Karls IV. vom 20. August 1357 in seiner Domherrenkurie angefertigt wurde ${ }^{25}$. Am 22. März 1429 ist er als Domherr sowie als einer der Testamentsvollstrecker des ein paar Jahre zuvor verstorbenen ermländischen Dekans Bartholomäus Boreschows belegt ${ }^{26}$. Im April 1430 wird er unter den Mitgliedern des Kapitels erwähnt ${ }^{27}$. In den Kapitelakten erscheint er auch im Jahre $1431^{28}$. Am 19. Januar 1436 wird er als Domherr und Stadtrichter in Frauenburg genannt. Auf diesen Tag ist auch sein Brief an den Stadtrat Danzigs datiert, in welchem er im Namen eines ungenannten Untertanen des Kapitels darum bittet, diesem Rechtshilfe zu gewähren $^{29}$. Am 13. Februar dieses Jahres trat er als ermländischer Domherr in Elbing auf ${ }^{30}$. Zusammen mit anderen zu diesem Zeitpunkt in Frauenburg residierenden Domherren kann man ihn sicher auch am 11. März 1438 nachweisen ${ }^{31}$. An der Frauenburger Domkirche ist er auch am 18. Mai 1439 belegt $^{32} .1441$ erscheint er in den Quellen als Kapiteladministrator in Allen-

21 Borawska, Kalle Jan, S. 108. Dass Johann Kalle in den ermländischen Quellen seit 1425 belegt ist, führte - sicherlich Borawska zufolge - auch Jurkowlaniec an, siehe idem, Nagrobki, S. 177.

22 Siehe CDW 4, Nr. 45; siehe auch V. Röhrich, Die Kolonisation des Ermlandes, in: Zeitschrift für die Geschichte und Alterthumskunde Ermlands (weiter als: ZGAE), 21 (1923), S. 395-396.

23 Siehe CDW, hg. v. C. P. Woelky, 3 (1874), Nr. 590; siehe auch Röhrich, Die Kolonisation, in: ZGAE 13 (1901), S. 855-856.

24 CDW 3, Nr. 593.

25 GStA PK, XX. HA, Perg. Urk., Schubl. XLVIII Nr. 7; ibid., OF 117, S. 59-64; vgl. CDW 4, Nr. 614; siehe auch ibid., Nr. 128.

26 CDW 4, Nr. 265.

27 Ibid., Nr. 311; vgl. auch GStA PK, XX. HA, OF 80b, S. 95-98.

28 CDW 4, Nr. 393.

29 Archiwum Państwowe w Gdańsku, Dokumenty Miasta Gdańska, 300D/42, Nr. 17.

30 GStA PK, XX. HA, OF 96, K. 84r-86r.

31 AAWO, Dok. Kap. S 28/2.

32 AAWO, Dok. Kap. H1/11. 
stein $^{33}$. Es ist auch zu bemerken, dass er am 7. Januar dieses Jahres in den Kapitelurkunden ausschließlich im Zusammenhang mit dem Kanonikat bezeugt ist. Dass er zu diesem Zeitpunkt aus der Kapitelkasse Mittel für den Erwerb von Vieh für das Kapitelvorwerk in Allenstein erhielt, kann davon zeugen, dass er schon damals die Pflichten des Administrators erfüllte, und zwar in der Kapitelburg in Allenstein ${ }^{34}$. Administrator war er zweifelsohne auch im Jahre $1442^{35}$. In Frauenburg findet man ihn am 21. April $1444^{36}$. Am 8. Januar 1445 erwähnt man ihn unter den beschenkten Personen im Testament des Küsters Arnold Huxers ${ }^{37}$. Im Zusammenhang mit dem besagten Testament ist er auch am 28. Februar dieses Jahres belegt ${ }^{38}$. Am 8. Februar 1446 schloss er zusammen mit dem Kapitel einen Vergleich mit den Frauenburger Ratsherren bezüglich des Stadthafens ${ }^{39}$. Am 14. Mai dieses Jahres war er Zeuge bei den Rechtstätigkeiten der Testamentsvollstrecker Arnold Huxers, der ein paar Tage zuvor verstorben war ${ }^{40}$. Er lebte sicherlich noch am 14. August 1447, als er einer der Zeugen eines Notariatsinstruments $\operatorname{war}^{41}$.

33 AAWO, Dok. Kap. L 83a; siehe auch SRW 1, S. 302. Eine solche Information finden wir auch in der Fachliteratur, siehe Borawska, Kalle Jan, S. 108; Jurkowlaniec, Nagrobki, S. 177.

34 AAWO, Acta Cap. S 1, K. 52v. Mehr zu dieser Quelle siehe auch F. Liedtke, Der Glockenturm des Domes zu Frauenburg und seine Glocken, in: ZGAE 15 (1905), S. 715, Anm. 3.

35 AAWO, Dok. Kap. L 83a; siehe auch die folgende Urkunde, die ihn zu diesem Zeitpunkt im Amt des Administrators belegt: GStA PK, XX. HA, Ordensbriefarchiv (weiter als: OBA), Nr. 28409.

36 AAWO, AK I, T 15, Fol. 56; siehe auch ibid., AB, Eh 123.

37 F. Hipler, Domcustos Arnold Huxer und sein Testament vom 8. Januar 1445, in: Pastoralblatt für die Diözese Ermland, 23, 11 (1891), S. 127-131.

38 AAWO, Dok. Kap. V 17a; siehe auch ibid., AK I, T 5, K. 20.

39 Siehe S. Szybkowski, J. Włodarski, Rola i znaczenie dokumentu „Super proprietate portus in Frauenburg" $z 8$ lutego 1446 roku dla rozwoju portu fromborskiego, in: Komunikaty Mazursko-Warmińskie, 4 (2002), S. 519-525 (Edition der Urkunde auf S. 522-524, Übersetzung ins Polnische auf S. 524). Die richtige Signatur der veröffentlichten Urkunde ist AAWO, Dok. Kap. F 24. Siehe auch die Abschriften dieser Urkunde, die von den Herausgebern der Edition nicht berücksichtigt wurden: AAWO, AB, Ef 47; siehe auch ibid. $\mathrm{AB}$, Eh 139.

40 AAWO, AK I, T 7, Fol. 92.

41 GStA PK, XX. HA, Perg. Urk., Schubl. 90 Nr. 6-7; Liv-, Est- und Curländisches Urkundenbuch, begründet v. F. G. v. Bunge, fortgesetzt v. H. Hildebrand und nach ihm v. P. Schwartz, 10 (1896), Nr. 372 (Regest); siehe auch Borawska, Kalle Jan, S. 108. Vermutlich 
Bisher behauptete man in der Fachliteratur, dass er am 10. Januar 1448 nicht mehr lebte, also zu jenem Zeitpunkt, als ein anderer Geistlicher sein Kanonikat übernommen haben soll ${ }^{42}$. Es ist aber darauf hinzuweisen, dass sein Todesdatum in den unveröffentlichten Akten des ermländischen Kapitels unter dem 10. Januar 1448 eingetragen wurde ${ }^{43}$. Obwohl der ganze Inhalt seines Testaments unbekannt bleibt, erfahren wir aus dem Eintrag in den obigen Urkunden, dass er gewisse Summen zu Bauzwecken (pro fabrica) bestimmte ${ }^{44}$. Bis zum heutigen Tag wird in der Frauenburger Domkirche seine Grabplatte bewahrt, in die u. a. sein Todesdatum eingemeißelt wurde ${ }^{45}$. Wie aus dem Eintrag im Anniversarienbuch des Kapitels von 1592 hervorgeht, gedachten die ermländischen Domherren Kalles im November ${ }^{46}$.

Die obigen Angaben legen es nahe, dass wir es in diesem Falle eher mit zwei verschiedenen Personen zu tun haben, weswegen die vom Autor des rezensierten Buches aufgestellte These eher für allzu kühn zu halten ist.

beglaubigte er die Urkunden noch am Ende dieses Jahres, siehe Röhrich, Die Kolonisation, in: ZGAE 21 (1923), S. 326-327.

42 Siehe Borawska, Kalle Jan, S. 108. An diesem Tag soll die vakante Pfründe schon an Kaspar Buls verliehen worden sein, siehe E. Brachvogel, Kleine Beiträge, in: ZGAE 22 (1926), S. 159-160. Der verstorbene Kalle erschien in den päpstlichen Quellen auch in späterer Zeit, siehe RG, bearb. v. J. F. Abert. W. Deeters, 6 (1985), Nr. 4741 (2. März 1448 - vgl. BP 6, Nr. 134), 625 (11. April 1448). Bemerkenswert ist auch die schnelle Reaktion des Hochmeisters, der schon am 14. Januar Briefe aus Marienburg nach Rom sandte, um das vakante Kanonikat mit seinem Sekretär und Schreiber Johann Beutin zu besetzen, siehe GStA PK, XX. HA, OF 16, S. 828-830. Eine andere Urkunde des Hochmeisters (eigentlich sein Konzept) ist dagegen auf den 20. Januar 1448 datiert. Zu diesem Zeitpunkt beschloss der Hochmeister des Deutschen Ordens Konrad von Erlichshausen, der sich auf das Privileg von Papst Nikolaus V. berief, dem ermländischen Bischof und dem Kapitel Bartholomäus Liebenwald für das vakante Kanonikat zu präsentieren, siehe GStA PK, XX. HA, OBA, Nr. 9463. Von dem im Zusammenhang mit dem Tode Johanns vakanten ermländischen Kanonikat und den Versuchen, sie zu gewinnen, erfahren wir auch aus dem Brief des Breslauer Bischofs Piotr an den Hochmeister vom 1. April 1448, siehe GStA PK, XX. HA, OBA, Nr. 9492.

43 Vgl. AAWO, Acta Cap. S 1, K. 54v. Sein Todesdatum, 10. Januar 1448, nennt zuletzt Jurkowlaniec, siehe idem, Nagrobki, S. 177.

44 AAWO, Acta Cap. S 1, K. 54v.; siehe auch ibid., AB, Eh 41.

45 Siehe Jurkowlaniec, Nagrobki, S. 176-177 (dort detaillierte Informationen über die Grabplatte des Geistlichen zusammen mit Hinweisen auf die ältere Fachliteratur). Die erhaltene Grabplatte erwähnte früher schon u. a. Borawska, siehe eadem, Kalle Jan, S. 108.

46 SRW 1, S. 244-245. 


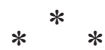

Ich möchte auch auf einige andere Fehler und Unzulänglichkeiten hinweisen, auf die ich bei der Buchlektüre stieß. Diesen Teil meines Beitrags beginne ich mit dem Hinweis auf die Information auf S. 46. P. Samól zufolge wurde die genannte Urkunde vom 8. Dezember 1344 „vom Pfarrer der Marienkirche in Danzig Konrad“ ausgefertigt. Unumstritten befand sich Konrad im Zeugenverzeichnis des besagten Diploms, doch ist es schwierig, daraus zu schließen, warum der Autor des rezensierten Buches behauptet, dass diese Urkunde eben von ihm ausgestellt wurde ${ }^{47}$.

Auf S. 85 stellte er zu Unrecht fest, dass Johann Krowel 1422 Pfarrer der Danziger St.-Katharinen-Kirche war. In diesem Jahr ist aber Nikolaus als Pfarrer der genannten Pfarrei belegt, der mit Nikolaus Kobelau identifiziert wird und die besagte Pfarrei mindestens bis 1442 innehatte ${ }^{48}$.

Weniger präzise, und zwar als Pfarrer aus Kulm, wurde darüber hinaus auf S. 282 Johannes Kopen bestimmt. Dieser Eintrag hätte bedeuten können, dass es sich hier um den Pfarrer einer lokalen Pfarrkirche handelte. Die Lektüre des Quelleneintrags, auf den der Autor rekurriert, bestätigt, dass er zu diesem Zeitpunkt Propst der Kulmer Benediktinerinnen war ${ }^{49}$.

Ein großes Verdienst des Autors ist unumstritten die Hervorhebung der in der bisherigen Fachliteratur unbeachteten Urkunde, in der im Zeugenverzeichnis Andreas Ruperti als Pfarrer der St.-Johannes-Kirche in Danzig erwähnt wird (S. 173). Im Gegensatz dazu, was P. Samól eher versehentlich notierte, erscheint Ruperti mit dieser Würde nicht 1422, sondern 1432 (am 13. August $)^{50}$. Wenn der Autor einige Seiten später (S. 174) auf den Pfarrer der Katharinenkirche in Danzig Nikolaus Kobelau hinweist, stellt er zu Recht fest, dass er in der Quelle 1432 als Rektor der St.-Katharinen-Pfarrkirche sowie Vikar und pommerellischer Offizial bezeichnet wird. Aber bei

47 Vgl. Preussisches Urkundenbuch, hg. v. M. Hein, 3 (1944), Nr. 692.

48 Siehe R. Krajniak, O średniowiecznych plebanach kościołów wchodzacych w skład obecnej Archidiecezji Gdańskiej na marginesie pracy Agnieszki Kobus, in: Zapiski Historyczne, 83, 3 (2018), S. 176-177.

49 Siehe Księgi Młodego Miasta Gdańska 1400-1455 [1458-1459], hg. v. K. Kopiński, P. Oliński, (2008), S. 25, Nr. 137 (der Autor beruft sich unrichtig auf Nr. 136).

50 Vgl. Deutschordenszentralarchiv Wien (weiter als: DOZA), Abteilung Urkunden, Nr. 3308. Zugang online (https://www.monasterium.net/mom/AT-DOZA/Urkunden/3308/charter) (05.07.2020). 
der Anführung des beglaubigenden Quelleneintrags begeht er einige Fehler in der Transkription ${ }^{51}$.

Erwähnenswert ist, dass P. Samól bei der Vorbereitung seiner Arbeit auch an die Quellen päpstlicher Provenienz anknüpfte, zumal er in seinem Buch einige Bände des Bullarium Poloniae zitiert. In der Einleitung (S. 30) bestätigt er, dass er sich auch mit einem sehr kritischen Artikel von Marek Daniel Kowalski vertraut machte, der sich sowohl mit der Konzeption als auch mit der Vorbereitung dieser Quellenedition auseinandersetzte ${ }^{52}$. Es scheint, dass P. Samól, der sich zwar der Unvollkommenheit des Bullarium bewusst ist und selbst annimmt, dass gewisse Datumsfehler in den zitierten Einträgen vorkommen können ${ }^{53}$, eher das dort gefundene Material hätte kontrollieren und möglicherweise auch auf das Repertorium Germanicum zurückgreifen sollen. Es ist eine Veröffentlichung, in der er nicht nur die in seiner Arbeit genutzten Quellen, sondern auch andere im Bullarium unveröffentlichte Einträge hätte finden können ${ }^{54}$. Obwohl ich nicht ganz

51 Die Transkription P. Samóls in Anm. 125 sieht wie folgt aus: „Nicolaus Cobelaw rector patrchialis ecalesie ad sanctam katherinam ante muros opidi Gdanem, vicarius In spenalibus et officialis“. Es scheint, dass der richtige Eintrag folgendermaßen lauten sollte: "Nicolaus Cobelau Rector parrochialis ecclesie ad sanctam katherinam antiquiorum opidi Gdanensis Vicarius In Spiritualibus et Officialis terre pomeranie“, vgl. DOZA, Abteilung Urkunden, Nr. 3308. Kleine Fehler in der Transkription bemerkt man auch in Anm. 127 auf S. 174; vgl. die Transkription des Autors und die Originalfassung: DOZA, Abteilung Urkunden, Nr. 3457, Zugang online https://www.monasterium.net/mom/AT-DOZA/ Urkunden/3457/charter (05.07.2020).

52 M. D. Kowalski, Uwagi o „Bullarium Poloniae“. Spojrzenie na koncepcje edycji i wykonanie, in: Studia Źródłoznawcze, 45 (2007), S. 71-97. Der Autor lässt dagegen die andere Arbeit von Kowalski aus, in der der letzte der herausgegebenen Bände des Bullarium, auch im rezensierten Buch zitiert, kritisch dargestellt wurde, siehe idem, Krytyczne uwagi o najnowszym, siódmym tomie „Bullarium Poloniae“, in: Roczniki Historyczne, 73 (2007), S. 214-231.

53 Siehe S. 185, Anm. 206 im rezensierten Buch.

54 Begründet wäre, die folgenden Quelleneinträge zu untersuchen: RG, bearb. v. G. Tellenbach, 2 (1933-1938), Sp. 308-309; 4, Sp. 557-558; 5, bearb. v. H. Diener, B. Schwarz, (2004), Nr. 319, 1475; 6, Nr. 990, 4741; 9, bearb v. H. Höing, H. Leerhoff, M. Reimann, (2000), Nr. 1379. Darüber hinaus fand sich in den vom Autor angeführten päpstlichen Quellen ein kleiner Fehler. Auf S. 172 in Anm. 114 sollte anstatt des Bd. 3 der Bd. 4 des Bullarium Poloniae angeführt werden. In einem Falle sollte der Autor zudem an die vollständige Quellenedition anknüpfen und anstatt „BP, Vol. 6, Nr. 335“ wie folgt zitieren: Vetera monumenta Poloniae et Lithuaniae gentiumque finitimarum historiam illustrantia maximam partem nondum edita ex tabulariis Vaticanis deprompta collecta ac serie chronologica disposita, 2: 1410-1572, hg. v. A. Theiner, (1861), Nr. 111. 
imstande bin, dies völlig sachkundig zu bewerten, ist die Urkunde vom 3. Dezember 1389, in der Andreas de Lewyn erscheint, am interessantesten. Er war damals Kapellan der St.-Georg-Kapelle außerhalb der Stadtmauern Danzigs ${ }^{55}$. Dass man sich in den Quellen päpstlicher Provenienz auch im Zusammenhang mit den Kircheninstitutionen, die zweifelsohne in der Jungstadt gelegen waren, der Bezeichnung ,außerhalb der Stadtmauern Danzigs" bediente, was übrigens der Autor selbst wiederholt ${ }^{56}$, ist von Bedeutung, und zwar wegen der vom Autor auf S. 155 geführten Erwägungen, dass es in der Jungstadt möglicherweise ein St.-Georg-Spital gab. Die Anknüpfung an diese Quelle wäre begründet, auch wenn sich die besagte Erwähnung, die in der bisherigen Geschichtsschreibung unbeachtet geblieben zu sein scheint, doch auf das St.-Georg-Spital (die gleichnamige Kapelle in der Altstadt Danzig) beziehen sollte, was sehr wahrscheinlich ist.

Einen kurzen Kommentar verdienen auch die Angaben, die von P. Samól auf S. 168 in Anm. 91 gemacht wurden. Er informiert dort über die Kirchenorganisation im patrimonium Kulms und beruft sich auf Tomasz Jasiński ${ }^{57}$. Dem Autor des rezensierten Buches zufolge knüpft der Posener Forscher in seiner Arbeit „an den Streit um die Gründung einer neuen Pfarrei in dem von der Stadt entfernten Sarnowo" an. Demnach soll Jasiński im besagten Buch behauptet haben, dass „die altstädtischen Pfarrer wegen dieses Streites darum bemüht waren, die Aussonderung einer neuen Pfarrei, die einen Teil des patrimoniums umfassen sollte, zu verhindern“. Die Lektüre des hier angeführten Abschnittes der Monografie von Jasiński zeigt aber eindeutig, dass sich der Streit auf den Bau einer neuen Pfarrkirche nicht in Sarnowo, sondern in Szynych bezog und nicht die altstädtischen Pfarrer, sondern der Pfarrer in Sarnowo diesen Beschluss zu bestreiten suchte ${ }^{58}$. Der Streit soll laut Jasiński vom Kulmer Offizial ca. 1340 gelöst worden sein (Samól nennt exakt das Jahr 1340). Am Rande sollte man noch hinzufügen, dass ich imstande war, dieses Datum präziser zu bestätigen. Dank den Untersuchungen zur Laufbahn der Kulmer Offiziale wissen wir, dass eine

\footnotetext{
55 RG 2, Sp. 474.

56 Siehe S. 161, Anm. 58 im rezensierten Buch.

57 Siehe T. Jasiński, Przedmieścia średniowiecznego Torunia i Chełmna, (1982), S. 50.

58 Vgl. ibid.
} 
entsprechende Urkunde frühestens im September 1341 angefertigt worden sein kann, weswegen dieses Datum zurzeit sehr wahrscheinlich ist ${ }^{59}$.

Die folgenden geringen Bemerkungen, die ich an dieser Stelle auch machen will, beziehen sich hauptsächlich auf die Redaktionsfragen. Zum ersten finde ich, dass der polnische Begriff pleban (Pfarrer bzw. Priester) eine viel besser begründete Bezeichnung für die mittelalterlichen Geistlichen anstatt proboszcz ist (so z. B. auf S. 42, 46, 56, 85, 168-170, 172-175). Im rezensierten Buch stoßen wir relativ häufig auch auf den Begriff państwo zakonu krzyżackiego w Prusach (Deutschordensstaat in Preußen). Hier bin ich der Meinung, dass die Bezeichnung władztwo zakonne (Deutschordensherrschaft) viel zutreffender und richtiger ist. Immer häufiger verwendet man darüber hinaus (obwohl dies vor vielen Jahren auch der Fall war) den Begriff zakon niemiecki (der Deutsche Orden), der eindeutig aus dem Quellenmaterial hervorgeht und trotz einer gewissen Geschichtsschreibungstradition die Bezeichnung zakon krzyżacki (Kreuzorden) verdrängen sollte. Unter den Unzulänglichkeiten findet man auch jene, die sich auf die Kopialbücher aus den Berliner Beständen (genau OF 3, 16 und 17) beziehen. P. Samól führt Kartenzahlen anstatt von Seitenzahlen an, und eben auf diese Weise erfolgt die Paginierung in den besagten Büchern ${ }^{60}$. Den Wert der erfolgreichen und wichtigen Arbeit vermindern leider fehlende Register. Trotz der obigen Bemerkungen kann die Publikation sehr positiv bewertet werden.

Übersetzt von

Liliana Lewandowska

59 Siehe R. Krajniak, Duchowieństwo kapituly katedralnej w Chełmży do 1466 roku. Studium prozopograficzne, (2013), S. 174-175.

60 Auf eine solche Situation stößt man auf S. 123 in Anm. 366 (hier zusätzlich unrichtige Seitenzahl - richtig S. 616-617), S. 124 in Anm. 372, S. 129 in Anm. 390, S. 132 in Anm. 409, S. 133 in Anm. 415, S. 134 in Anm. 431, S. 170 Anm. 107, S. 234 in Anm. 121, S. 251 in Anm. 243, S. 255 in Anm. 280 und 283, S. 269 in Anm. 377, S. 302 in Anm. 60-61 und 64, S. 303 in Anm. 66, S. 304 in Anm. 72 und 74. Richtig zitiert er dagegen eine andere Quelle OF 16, siehe S. 269, in Anm. 380 im rezensierten Buch. 


\section{O PRÓBIE IDENTYFIKACJI JOHANNA KALO, PLEBANA KOŚCIOŁA \\ PARAFIALNEGO POD WEZWANIEM ŚW. BARTŁOMIEJA W MŁODYM MIEŚCIE GDAŃSKU, Z KANONIKIEM WARMIŃSKIM JOHANNEM KALLE}

ORAZ KILKA INNYCH UWAG NA MARGINESIE NAJNOWSZEJ KSIĄŻKI

PIOTRA SAMÓLA

STRESZCZENIE

Celem artykułu jest krytyczna ocena książki Piotra Samóla pt. Młode Miasto Gdańsk (1380-1455) i jego patrymonium. Poza omówieniem treści tej monografii zasadniczą część artykułu stanowią dalsze uwagi. Bliższej analizie poddana została przede wszystkim próba utożsamienia przez autora recenzowanej pracy plebana kościoła parafialnego pw. św. Bartłomieja w Młodym Mieście Gdańsku Johanna Kalo, z noszącym to samo imię i podobne nazwisko kanonikiem warmińskim. W treści artykułu przedstawiono szczegółowo karierę kanonika warmińskiego Johanna Kalle, starając się wykazać, że nie mógł być on raczej tożsamy z żyjącym w tym samym czasie plebanem parafii młodomiejskiej.

VOM VERSUCH, JOHANN KALO, DEN PFARRER DER BARTHOLOMÄUSPFARRKIRCHE IN DER JUNGSTADT DANZIG, MIT DEM ERMLÄNDISCHEN DOMHERRN JOHANN KALLE GLEICHZUSETZEN

UND EINIGE ANDERE RANDBEMERKUNGEN ZUM NEUESTEN BUCH VON PIOTR SAMÓL

ZUSAMMENFASSUNG

Im Beitrag ziele ich auf eine kritische Bewertung des Buches Piotr Samóls unter dem Titel Młode Miasto Gdańsk (1380-1455) i jego patrymonium (Die Jungstadt Danzig (1380-1455) und ihre Besitzungen) ab. Neben der Darstellung des Inhalts dieser Monografie bilden die Bemerkungen den grundlegenden Teil des vorliegenden Artikels. Einer näheren Analyse unterzog ich vor allem den Versuch des Autors, Johann Kalo, den Pfarrer der Bartholomäuspfarrkirche in der Jungstadt Danzig, mit dem beinahe gleichnamigen ermländischen Domherrn gleichzusetzen. Ausführlich schildere ich die Laufbahn des ermländischen Domherrn Johann Kalle und wollte dadurch nachweisen, dass er mit dem zum selben Zeitpunkt lebenden Pfarrer der jungstädtischen Pfarrei nicht identisch sein kann. 


\title{
AbOUT THE ATTEMPT TO IDENTIFY JOHANN KALO, THE PARISH PRIEST OF THE PARISH CHURCH OF ST. BARTHOLOMEW IN THE YOUNG CITY OF GDAŃSK, WITH THE WARMIAN CANON JOHANN KALLE
}

\author{
A FEW OTHER REMARKS ON THE MARGINS OF THE MOST RECENT BOOK \\ BY PIOTR SAMÓL \\ SUMMARY
}

The aim of the article is to critically evaluate Piotr Samol's book Młode Miasto Gdańsk (1380-1455) i jego patrymonium (The Young City of Gdańsk (1380-1455) and its patrimony). Apart from discussing the content of this monograph, further remarks constitute the main part of the article. What the article analyzes is the attempt to identify the parish priest of the Church of St. Bartholomew in the Young City of Gdańsk Johann Kalo, with a Warmian canon bearing the same name and a similar surname. The article presents in detail the career of the Warmian canon, Johann Kalle, trying to show that he could not be identical with the parish priest of the Young Town of Gdańsk, who was living at the same time. Translated by Agnieszka Chabros

\section{SŁOWA KLUCZOWE / SCHLAGWORTE / KEYWORDS}

- średniowiecze; kler; Młode Miasto Gdańsk

- Mittelalter; Geistlichkeit; Jungstadt Danzig

- Middle Ages; clergy; Young City of Gdańsk

\section{BIBLIOGRAFIA / BIBLIOGRAFIE / BIBLIOGRAPHY}

\section{ŹRÓDłA ARCHIWALNE / ARCHIVALISCHE QUELLEN / ARCHIVAL SOURCES}

Archiwum Archidiecezji Warmińskiej w Olsztynie

- Archiwum Biskupie, Sign. Ef 47; Eh 41, 123, 139; H 295c.

- Acta Capitularia, S 1.

- Archiwum Kapituły, Sign. I, T 5; I, T 7; I, T 15.

- Dokumenty Kapituły, Sign. F 24; H1/11; L 83a; S 28/2; V 17a.

Archiwum Państwowe w Gdańsku, Dokumenty Miasta Gdańska, Sign. 300D/42, Nr. 17.

Deutschordenszentralarchiv Wien, Abteilung Urkunden, Nr. 3308, 3457.

Geheimes Staatsarchiv Preußischer Kulturbesitz Berlin-Dahlem, XX. Hauptabteilung

- Ordensbriefarchiv, Nr. 9463, 9492, 28409;

- Ordensfolianten, Nr. 16; 80b; 96; 117;

- Pergament-Urkunden, Schublade 90, Nr. 6-7; XLVIII Nr. 7. 


\section{ŹRÓDŁA DRUKOWANE / GEDRUCKTE QUELLEN / PRINTED SOURCES}

Album seu matricula juridicae Universitatis Pragensis ab anno Christi 1372 usque ad annum 1418, 1 (Monumenta historica Universitatis Carolo-Ferdinandae Pragensis 2, 1834).

Bullarium Poloniae litteras apostolicas aliaque monumenta Poloniae Vaticana continens, 6: 1447-1464, hg. v. I. Sułkowska-Kuraś, S. Kuraś, (1998).

Codex diplomaticus Warmiensis oder Regesten und Urkunden zur Geschichte Ermlands, hg.v. C. P. Woelky, 3 (1874) und hg. v. V. Röhrich, F. Liedtke, H. Schmauch, 4 (1935).

Hipler F., Domcustos Arnold Huxer und sein Testament vom 8. Januar 1445, in: Pastoralblatt für die Diözese Ermland, 23, 11 (1891), S. 126-131.

Księgi Młodego Miasta Gdańska 1400-1455 (1458-1459), hg. v. K. Kopiński, P. Oliński, (2008).

Liber decanorum facultatis philosophicae Universitatis Pragensis ab anno Christi 1367 usque ad annum 1585, 1 (Monumenta historica Universitatis Carolo-Ferdinandae Pragensis 1, 1830).

Liv-, Est- und Curländisches Urkundenbuch, begründet v. F. G. v. Bunge, fortgesetzt v. H. Hildebrand und nach ihm v. P. Schwartz, 10 (1896).

Die Matrikel der Universität Wien, 1: 1377-1450, (1954).

Preussisches Urkundenbuch, hg. v. M. Hein, 3 (1944).

Repertorium Germanicum. Verzeichnis der in den päpstlichen Registern und Kameralakten vorkommenden Personen, Kirchen und Orte des Deutschen Reiches, seiner Diözesen und Territorien vom Beginn des Schismas bis zur Reformation, bearb. v. G. Tellenbach, 2 (1933-38); bearb. v. U. Kühne, 3 (1935); bearb. v. K. A. Fink, 4 (1943-1958); bearb. v. H. Diener und. B. Schwarz, 5 (2004); bearb. v. J. F. Abert und W. Deeters, 6 (1985); bearb. v. H. Höing, H. Leerhoff und M. Reimann, 9 (2000).

Scriptores rerum Warmiensium oder Quellenschriften zur Geschichte Ermlands, hg. v. C. P. Woelky, J. M. Saage, 1 (1866).

Vetera monumenta Poloniae et Lithuaniae gentiumque finitimarum historiam illustrantia maximam partem nondum edita ex tabulariis Vaticanis deprompta collecta ac serie chronologica disposita, 2: 1410-1572, hg. v. Augustinus Theiner, (1861).

\section{LITERATURA / LITERATUR / LITERATURE}

Bogdan D., Przeracki J., Urzędnicy Starego i Nowego Miasta Braniewa do 1772 roku, (2018). Borawska T., Kalle Jan, in: Słownik biograficzny kapituły warmińskiej, bearb. v. T. Borawska, M. Borzyszkowski, A. Kopiczko, J. Wojtkowski, hg. v. J. Guzowski, (1996), S. 108.

Brachvogel E., Kleine Beiträge, in: Zeitschrift für die Geschichte und Alterthumskunde Ermlands, 22 (1926), S. 151-165.

Freytag H., Die Beziehungen der Universität Leipzig zu Preussen von ihrer Begründung bis zur Reformation 1409-1539, in: Zeitschrift des Westpreussischen Geschichtsvereins, 44 (1902), S. 1-158.

Jasiński T., Przedmieścia średniowiecznego Torunia i Chełmna, (1982).

Jurkowlaniec T., Nagrobki średniowieczne w Prusach, (2015).

Kenkel H., Studenten aus Ost- und Westpreußen an außerpreußischen Universitäten vor 1815, (1981). 
Kowalski M. D., Krytyczne uwagi o najnowszym, siódmym tomie „Bullarium Poloniae“, in: Roczniki Historyczne, 73 (2007), S. 214-231.

Kowalski M. D., Uwagi o „Bullarium Poloniae“. Spojrzenie na koncepcję edycji i wykonanie, in: Studia Źródłoznawcze, 45 (2007), S. 71-97.

Krajniak R., Duchowieństwo kapituły katedralnej w Chełmży do 1466 roku. Studium prozopograficzne, (2013).

Krajniak R., O średniowiecznych plebanach kościołów wchodzących w skład obecnej Archidiecezji Gdańskiej na marginesie pracy Agnieszki Kobus, in: Zapiski Historyczne, 83, 3 (2018), S. 173-191.

Liedtke F., Der Glockenturm des Domes zu Frauenburg und seine Glocken, in: Zeitschrift für die Geschichte und Alterthumskunde Ermlands, 15 (1905), S. 705-720.

Prussia scholastica. Die Ost- und Westpreussen auf den mittelalterlichen Universitäten, gesammelt v. M. Perlbach, (1895).

Röhrich V., Die Kolonisation des Ermlandes, in: Zeitschrift für die Geschichte und Alterthumskunde Ermlands, 13 (1901), S. 742-980 und 21 (1923), S. 394-411.

Ruciński R., Środowiska intelektualne wielkich miast pruskich $w$ średniowieczu, (2005) (Manuskript der Dissertation).

Samól P., Młode Miasto Gdańsk (1380-1455) i jego patrymonium, (2018).

Szybkowski S., Włodarski J., Rola i znaczenie dokumentu „Super proprietate portus in Frauenburg" $z 8$ lutego 1446 roku dla rozwoju portu fromborskiego, in: Komunikaty Mazursko-Warmińskie, 4 (2002), S. 519-525.

Tř́ška J., Životopisný slovník predhusitské pražské univerzity 1348-1409, (1981). 